\title{
Painful diabetic peripheral neuropathy
}

\section{Health care costs and complications from 2010 to 2015}

\author{
Musa Kiyani, BS, Zidanyue Yang, MB, Lefko T. Charalambous, BS, Syed M. Adil, BS, Hui-Jie Lee, PhD, \\ Siyun Yang, MS, Promila Pagadala, PhD, Beth Parente, PA-C, MHS, Susan E. Spratt, MD, \\ and Shivanand P. Lad, MD, PhD
}

Correspondence

Dr. Lad

nandan.lad@duke.edu

Neurology: Clinical Practice February 2020 vol. 10 no. 1 47-57 doi:10.1212/CPJ.0000000000000671

\begin{abstract}
Objective

To quantify health care resource utilization and risk of complications in painful diabetic peripheral neuropathy (pDPN).

\section{Methods}

Adult patients diagnosed with diabetes mellitus or diabetic peripheral neuropathy (DPN) were identified in MarketScan from January 2010 to December 2015. Subgroups (pDPN and nonpainful DPN) were based on the use of pain medications 6 months before a new indexed diagnosis and 1 year thereafter. Health care costs were collected for up to 5 years, and complications charted for those with at least 1 and 2 years of follow-up. Complication comparisons were made using $\chi^{2}$ or Fisher exact tests, and a multivariable regression cost model was fit with log link function using generalized estimating equations.
\end{abstract}

\section{Results}

Among 360,559 patients with diabetes (62 \pm 14 years; 54.3\% female), 84,069 (23.3\%) developed pDPN, 17,267 (4.8\%) experienced nonpainful DPN, and the majority (259,223, $71.9 \%)$ were controls with diabetes without neuropathy. At baseline, costs associated with pDPN patients were $20 \%$ higher than diabetic controls (95\% confidence interval [CI] [1.19, $1.21], p<0.001$ ), which increased to $31 \%$ in the 5 th year (95\% CI $[1.27,1.34], p<0.001$ ). Patients with pDPN had 200\%, 356\%, and $224 \%$ of the odds of using opioids, anticonvulsants, and antidepressants, respectively, compared with diabetic controls. The amputation risk in the pDPN subgroup was 16.24 times that of diabetic controls (95\% CI [2.15, 122.72], $p=0.0003$ ), and $87 \%$ more patients with pDPN experienced lower extremity infections (95\% CI [1.43, $2.46], p<0.0001$ ) within a year. Within 2 years, $2.2 \%$ of patients with pDPN had falls and fallrelated injuries compared with $1.1 \%$ of diabetic controls $(p<0.0001)$.

\section{Conclusions}

Our study characterizes a substantial pDPN cohort in the United States, demonstrating considerable morbidity and economic costs.
Diabetic peripheral neuropathy (DPN) is a frequent chronic complication of diabetes that can lead to pain, tingling, numbness, weakness, foot ulceration, Charcot arthropathy, and even amputation of the lower limbs. ${ }^{1}$ Among patients with diabetic mellitus (DM), approximately 
$8 \%$ may present with neuropathy at the time of diagnosis, and about half of them will develop neuropathy within 25 years. $^{2}$ A community-based study in the United Kingdom spanning over 4 years found that a third of patients with diabetes have painful diabetic peripheral neuropathy $(\mathrm{pDPN})$, regardless of their neuropathic deficits. ${ }^{3}$

If inadequately treated, $\mathrm{pDPN}$ can persist and lead to debilitating chronic pain, affecting mood, sleep, and quality of life. ${ }^{4,5}$ Ultimately, treatment relies on better glycemic control and symptom amelioration with tricyclic antidepressants and anticonvulsants. ${ }^{6}$ Although narcotics are commonly used for nociceptive pain, recent retrospective studies on $\mathrm{pDPN}$ have shown their widespread use for neuropathic pain as well. ${ }^{7}$

Previous retrospective studies show that 1-year excess health costs for pDPN, beyond diabetes, can be as high as $\$ 8500$. $^{8}$ However, the objective assessment of pDPN has been difficult, with researchers relying wholly on diagnostic codes without emphasizing concurrent neuropathic pain medication usage. Acute, rather than chronic, costs have been emphasized as well. We have used data from a large database to perform this longitudinal retrospective study to quantify the effect of $\mathrm{pDPN}$ on the US health care system, its downstream complications (foot infections and amputations), and nonmedical management (e.g., use of chiropractic and physical therapy).

\section{Methods}

\section{Data retrieval}

Data on patients aged at least 18 years who were first diagnosed with DM (International Classification of Diseases, Ninth Revision, Clinical Modification [ICD-9-CM] diagnosis codes 250.xx) or DPN (ICD-9-CM diagnosis codes 250.6x and 357.2) between January 2010 and December 2015 were analyzed using the Truven Health MarketScan Commercial Claims and Encounters and Medicare Supplemental and Coordination of Benefits databases (figure 1). Patients with DM experiencing serious morbidities, such as ketoacidosis and hyperosmolar hyperglycemic states (250.1x, 250.2, and 250.3), were excluded. The index date for DM controls without DPN diagnosis was defined as the first diagnosis date of diabetes, and for patients with DPN, it was the first diagnosis date of DPN.

For patients with DPN diagnosis, we further investigated the prescriptions of pain medications and classified patients with at least 1 refill of pain medications during the 6-month preindex and 1-year postindex period as the pDPN cohort. Patients with DPN diagnosis but without pain medication prescriptions throughout the 6-month preindex and 1-year postindex period were considered as the nonpainful DPN cohort. In summary, patients with $\mathrm{pDPN}$ were a subset of patients with diabetes and diabetic peripheral neuropathy who used pain medications during a predetermined time window, patients with nonpainful DPN were the remaining patients with DPN, whereas the last remaining cohort of patients with diabetes without neuropathy diagnostic codes or pain medication usage were labeled as "diabetic controls".

Pain medications used in cohort identification were opiate agonists (MarketScan therapeutic classes 60 and 61, including opiate partial agonists), antiepileptic drugs (classes 65-68, such as hydantoin derivatives and succinimides), and antidepressants (class 69, including selective serotonin reuptake inhibitors and serotonin-norepinephrine reuptake inhibitors). Table e-1 (links.lww.com/CPJ/ A103) helps show the small but similar number of patients with $\mathrm{pDPN}$ and DM with concurrent diagnoses of seizures, epilepsy, or migraines that were not excluded from further analyses.

An alternative method to identify patients with pDPN using a combination of DM, DPN, and chronic pain diagnoses was also considered. Using such a method, however, resulted in only 1075 patients with pDPN fulfilling both criteria (i.e., chronic pain diagnosed at the time of DPN or anytime thereafter and analgesic use within a year of DPN diagnosis), indicating that unfortunately, 2 different cohorts were being identified. To reduce the risk of identifying important "false positives," this alternative identification method was abandoned.

We used a 1-month prediagnosis "washout" period to reduce the influence of escalating hospital visits and costs before a DM or DPN diagnosis and used the previous year to assess comorbidities. For health care resource utilization (HCRU) analysis, only those with continuous enrollment in every month throughout their 1-year pre- and post-diagnosis period were chosen; continuous enrollment throughout 5 years of followup was not a requirement for subsequent analyses. However, each patient's annual service and medication data were retained only if they had continuous enrollment in all months of that year. We also ensured that all pharmacy claims were fully captured by MarketScan to avoid underestimating medication costs and incidence rates identified using prescriptions. Those without any medication refills were excluded to avoid underestimation of medication usage and costs.

The relative incidence of downstream complications in patients with pDPN at 1- and 2-year follow-up was calculated. These were defined using a combination of diagnostic and procedural codes for lower limb amputations (ICD-9: 84.3, 84.10-84.12, 84.14, 84.15, 85.17, and 84.91; CPT: 27590-27592, 27594, 27596, 27599, 27880-27882, 27888, $27889,28800,28805,28810,28820$, and 28825), lower extremity infections (ICD-9: 680.7, 681.1, 681.9, 682.7, 686.0, 686.1, 686.8, 686.9, 707.1, 707.8, 707.9, 728, 729.1, $729.4, \quad 730.06-730.09, \quad 730.16-730.19, \quad 730.26-730.29$, 730.36-730.39, 730.86-730.89, 730.96-730.99, and 785.4), and falls and fall-related injuries (E880.0, E880.1, E880.9, and E884.2-E884.6). 
Figure 1 Flowchart indicating data retrieval steps, study outcomes, and patient numbers using the Truven Health MarketScan Medicare and Commercial databases

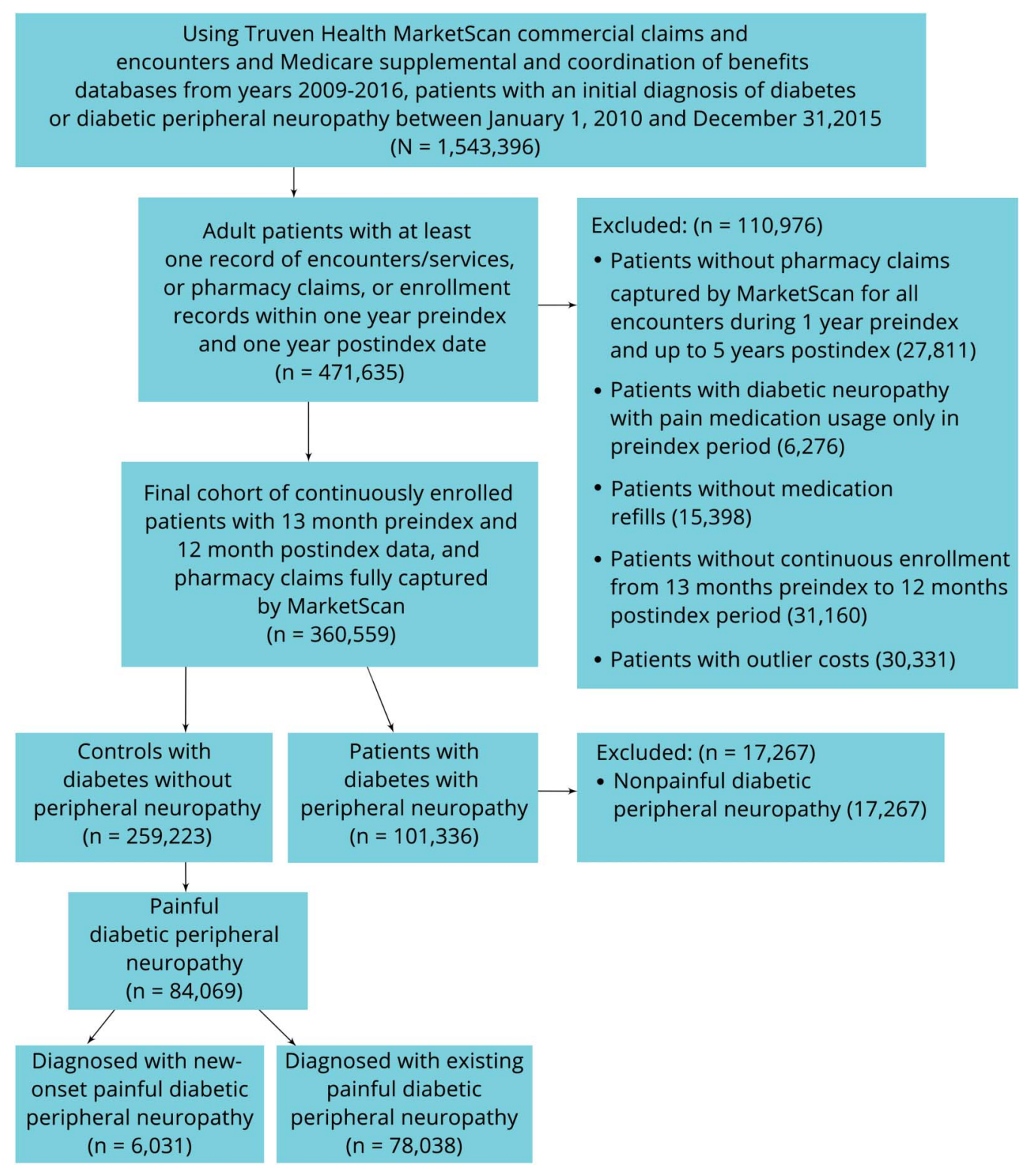

The relative incidence of nonmedical management in patients with pDPN at 1- and 2-year follow-up periods was calculated via MarketScan's procedure groups (PROCGRP). These included physical medicine (groups 181-189), chiropractic therapy (group 195), psychological counseling (groups 135-137 and 139), and home health physical, occupational, and speech therapy (group 478).

\section{Statistical analysis}

Continuous variables were summarized using means/ medians, SDs, interquartile ranges (IQRs), and ranges. Categorical variables were presented as frequency counts and percentages and compared among groups using the $\chi^{2}$ test or
Fisher exact test. Baseline characteristics (e.g., age, sex, employment status, insurance status, and residence geographic region) were defined at the index event. Baseline DeyoCharlson comorbidity scores were determined using outpatient and inpatient service records during the 1-year preindex time window with a 30-day washout period. ${ }^{9,10}$

HCRU was calculated as annual total cost after index date measured at 1, 2, 3, 4, and 5 years, including outpatient medication costs (from outpatient pharmacy claims) and inpatient and outpatient service costs. Baseline costs were again defined as costs in the 1-year preindex time with a 30day washout period. The top and bottom $1 \%$ of inpatient and 
outpatient payments and the top $2 \%$ of medication payments were considered outliers, and these patients were excluded from further HCRU analysis. Patients with negative medication payments (constituting $<1 \%$ of the data set) were also excluded. Costs were adjusted to 2017 US dollars using inflation rates from the Bureau of Labor Statistics Consumer Price Index.

A multivariable regression model with log link using generalized estimating equations (GEEs) was fit to compare the costs longitudinally across disease groups ( $\mathrm{pDPN}$, nonpainful DPN, and DM controls). Each patient was treated as an individual cluster to account for within-subject correlation. Autoregressive correlation structure was chosen as a working correlation matrix based on the quasi-likelihood under the independence model criterion. Patients who had zero cost at baseline were adjusted to $\$ 1$ so that they could be included in the GEE model. The following variables were accounted for in this multivariable model: disease group, time, disease group and time interaction, patient age at index date, sex, insurance type (Commercial vs Medicare), region, and Charlson comorbidity index. The level of statistical significance was set at alpha $=0.05$. Analyses were conducted using SAS software version 9.4 (SAS Institute Inc., Cary, NC).

\section{Standard protocol approvals, registrations, and patient consents}

Data obtained via the MarketScan database were deidentified and complied with the Health Insurance Portability and Accountability Act. This study was approved as an exempt study by the Duke Institutional Review Board.

\section{Data availability}

Anonymized data not explicitly published within this article were retrieved from a commercial database (MarketScan) and might be possibly shared by request from a qualified investigator.

\section{Results}

\section{Disease prevalence}

Our retrospective MarketScan analysis revealed a total of 360,559 adult patients first diagnosed with diabetes or DPN from 2010 to 2015. Among these, 84,069 (23.3\%) developed pDPN, 17,267 (4.8\%) developed nonpainful DPN, and the majority $(259,223,71.9 \%)$ were DM controls without any neuropathy. The prevalence of the 3 disease groups was largely stable over the 6 years of the study.

\section{Demographics}

Table 1 summarizes the baseline demographic information of the 360,559 patients first diagnosed with DM or DPN during 2010-2015. All patients were followed for up to 5 years with a median follow-up time of 856 days $($ mean $=779$
Overall, patients with pDPN tended to accrue the highest costs in all components of the total health care costs.

days, $\mathrm{SD}=535.5$ days, $\mathrm{IQR}=419-1037$ days). Briefly, $\mathrm{DM}$ controls and patients with pDPN tended to have a slight female predominance (55.8\% and $52.7 \%$, respectively), whereas patients with nonpainful DPN were predominantly men $(60.3 \%$ vs $39.7 \%$ women $)$. Patients with pDPN, compared with DM controls, also tended to be more commonly older and on Medicare (median ages were 66 years and 60 years for $\mathrm{pDPN}$ and DM controls, respectively). Compared with $39.6 \%$ of patients with pDPN, only $9.6 \%$ of DM controls had a Charlson comorbidity index higher than 2 . For the pDPN cohort, the median of baseline total costs (including inpatient/outpatient service costs and outpatient medication costs) was $\$ 7252.8$ or approximately $\$ 3,300$ higher than DM controls.

\section{Health care costs}

The total inpatient and outpatient service costs and total outpatient medication-related costs are aggregated by year and disease group in table 2. The outcome time trend is plotted in figure 2. Overall, patients with pDPN tended to accrue the highest costs in all components of the total health care costs. In addition, the time trend plots have similar patterns for all 3 disease groups, with costs escalating in the short term during the 1st year postdiagnosis and then decreasing gradually over time.

The pDPN cohort had double the costs of DM controls throughout our study time frame. The median costs for pDPN reached their peak (approximately $\$ 16,795$ ) in the 1st year after diagnosis, approaching almost $\$ 8,000$ above costs associated with DM or nonpainful DPN. While comparing nonpainful DPN and DM controls, the gap in median costs dropped to less than $\$ 200$ in the 1st year after diagnosis, but then widened with time. All 3 disease groups experienced the highest costs in the 1st year after diagnosis, stabilizing thereafter until the 4th year, and increasing slightly thereafter in the 5 th year.

Overall costs were split into outpatient medications costs and hospital services charges. We found that among the 360,559 identified patients, patients with pDPN spent more than 3 times as much on medications as DM controls at baseline. The highest median expenditure of $\$ 5188.3$ occurred in the 1st year post-pDPN diagnosis at a time when DM controls had medication expenditures of \$1726.6 and nonpainful DPN of \$2763.6. Inpatient and outpatient service costs followed a similar temporal trend where the 
Table 1 Patient demographics by disease group

\begin{tabular}{|c|c|c|c|c|}
\hline & Diabetics $(N=259223)$ & Nonpainful DPN (N = 17267) & pDPN (N = 84069) & Total $(\mathrm{N}=360559)$ \\
\hline \multicolumn{5}{|l|}{ Diagnosis year } \\
\hline 2010 & $26204(10.1 \%)$ & $1302(7.5 \%)$ & $8240(9.8 \%)$ & 35746 (9.9\%) \\
\hline 2011 & $42783(16.5 \%)$ & 2245 (13.0\%) & $14094(16.8 \%)$ & $59122(16.4 \%)$ \\
\hline 2012 & 35391 (13.7\%) & $2373(13.7 \%)$ & $13712(16.3 \%)$ & $51476(14.3 \%)$ \\
\hline 2013 & 26844 (10.4\%) & 2159 (12.5\%) & $10244(12.2 \%)$ & 39247 (10.9\%) \\
\hline 2014 & 72985 (28.2\%) & 5477 (31.7\%) & $21591(25.7 \%)$ & $100053(27.7 \%)$ \\
\hline 2015 & $55016(21.2 \%)$ & 3711 (21.5\%) & $16188(19.3 \%)$ & 74915 (20.8\%) \\
\hline \multicolumn{5}{|l|}{ Age at index event } \\
\hline $\mathbf{N}$ & 259223 & 17267 & 84069 & 360559 \\
\hline Mean (SD) & $60.6(14.3)$ & $69.6(12.7)$ & $66.2(12.5)$ & $62.3(14.1)$ \\
\hline Median (Q1, Q3) & $60.0(52.0,71.0)$ & $71.0(61.0,79.0)$ & $66.0(58.0,76.0)$ & $61.0(53.0,73.0)$ \\
\hline \multicolumn{5}{|l|}{ Sex } \\
\hline Male & 114572 (44.2\%) & $10411(60.3 \%)$ & 39745 (47.3\%) & $164728(45.7 \%)$ \\
\hline Female & 144651 (55.8\%) & 6856 (39.7\%) & $44324(52.7 \%)$ & $195831(54.3 \%)$ \\
\hline \multicolumn{5}{|l|}{ Insurance } \\
\hline Commercial & $159798(61.6 \%)$ & $5578(32.3 \%)$ & $35712(42.5 \%)$ & $201088(55.8 \%)$ \\
\hline Medicare & 99425 (38.4\%) & $11689(67.7 \%)$ & 48357 (57.5\%) & $159471(44.2 \%)$ \\
\hline \multicolumn{5}{|l|}{ Region } \\
\hline Northeast region & $54670(21.1 \%)$ & 3960 (22.9\%) & $12758(15.2 \%)$ & 71388 (19.8\%) \\
\hline North central region & $76583(29.5 \%)$ & 5794 (33.6\%) & $28356(33.7 \%)$ & $110733(30.7 \%)$ \\
\hline South region & $94851(36.6 \%)$ & $5050(29.2 \%)$ & 32087 (38.2\%) & $131988(36.6 \%)$ \\
\hline West region & 32370 (12.5\%) & 2432 (14.1\%) & $10694(12.7 \%)$ & $45496(12.6 \%)$ \\
\hline Unknown region & 749 (0.3\%) & $31(0.2 \%)$ & $174(0.2 \%)$ & $954(0.3 \%)$ \\
\hline \multicolumn{5}{|l|}{ Employment status } \\
\hline Full time/part time & 99875 (38.5\%) & 3279 (19.0\%) & $19289(22.9 \%)$ & $122443(34.0 \%)$ \\
\hline Retiree & $109582(42.3 \%)$ & $10970(63.5 \%)$ & $47050(56.0 \%)$ & $167602(46.5 \%)$ \\
\hline Other & $48221(18.6 \%)$ & $2965(17.2 \%)$ & $16975(20.2 \%)$ & $68161(18.9 \%)$ \\
\hline Long-term disability & $1545(0.6 \%)$ & $53(0.3 \%)$ & $755(0.9 \%)$ & $2353(0.7 \%)$ \\
\hline \multicolumn{5}{|l|}{ Deyo-Charlson Index } \\
\hline 0 & 153939 (59.4\%) & $1375(8.0 \%)$ & $5284(6.3 \%)$ & $160598(44.5 \%)$ \\
\hline 1 & $47318(18.3 \%)$ & $5360(31.0 \%)$ & $25983(30.9 \%)$ & $78661(21.8 \%)$ \\
\hline 2 & $33340(12.9 \%)$ & $4000(23.2 \%)$ & $19543(23.2 \%)$ & $56883(15.8 \%)$ \\
\hline$>2$ & $24626(9.5 \%)$ & $6532(37.8 \%)$ & $33259(39.6 \%)$ & $64417(17.9 \%)$ \\
\hline \multicolumn{5}{|c|}{$\begin{array}{l}\text { Total inpatient/outpatient cost at } \\
\text { baseline }\end{array}$} \\
\hline Mean (SD) & $8252.0(15480.2)$ & $8153.1(16480.0)$ & $13141.8(22344.0)$ & $9387.4(17492.5)$ \\
\hline Median (Q1, Q3) & $3916.3(1314.9,9370.4)$ & $3873.5(1595.8,8545.3)$ & $7252.8(3154.9,15063.0)$ & $4579.1(1622.8,10667.7)$ \\
\hline
\end{tabular}


Table 1 Patient demographics by disease group (continued)

\begin{tabular}{|c|c|c|c|c|}
\hline & Diabetics $(\mathrm{N}=259223)$ & Nonpainful DPN $(\mathrm{N}=17267)$ & pDPN (N = 84069) & Total $(\mathrm{N}=360559)$ \\
\hline \multicolumn{5}{|c|}{ Outpatient medication cost at baseline } \\
\hline Mean (SD) & 2930.3 (5197.9) & $3753.0(4801.4)$ & $6251.2(6786.3)$ & $3744.0(5762.6)$ \\
\hline Median (Q1, Q3) & $1367.9(352.1,3543.2)$ & $2416.0(550.6,5285.1)$ & $4485.9(1983.4,8243.0)$ & $1942.4(515.7,4771.8)$ \\
\hline \multicolumn{5}{|l|}{ Pain meds cost } \\
\hline Mean (SD) & $504.3(1780.0)$ & $14.5(120.4)$ & $831.2(2091.4)$ & $557.1(1825.4)$ \\
\hline Median (Q1, Q3) & $29.8(0.0,275.5)$ & $0.0(0.0,0.0)$ & $182.3(40.7,788.7)$ & $45.3(0.0,352.0)$ \\
\hline \multicolumn{5}{|c|}{ Follow-up days after index } \\
\hline Mean (SD) & $994.4(501.7)$ & $956.6(451.4)$ & $994.3(490.1)$ & $992.5(496.8)$ \\
\hline Median (Q1, Q3) & $853.0(611.0,1259.0)$ & $854.0(614.0,1156.0)$ & $867.0(611.0,1267.0)$ & $856.0(611.0,1256.0)$ \\
\hline
\end{tabular}

Abbreviations: DPN = diabetic peripheral neuropathy; pDPN = painful diabetic peripheral neuropathy.

Full time/part time: active full time and active part time.

Retiree: early retiree, Medicare-eligible retiree, and retiree (status unknown).

Other: COBRA continuee, surviving spouse/dependent, and other/unknown.

pDPN cohort had the highest cost burden throughout the study time frame.

Looking specifically at pain medications, as expected, patients with nonpainful DPN had a mean cost of $\$ 0$ in the 1 st year of diagnosis. Subsequently, the means were above 0 , indicating either that some do evolve into patients experiencing painful neuropathy or that their pain becomes unbearable without treatment. Because our study did not intend to capture this switch between disease groups, patients initially diagnosed as nonpainful DPN stayed in their respective cohort. Note, however, that the median costs for this nonpainful DPN group were still $\$ 0$ up to the 5th year after diagnosis, confirming our earlier definition that this is a cohort of patients with DPN who rarely take pain medications. Compared with DM controls, patients with pDPN spent much more on pain medications with the highest annual expenditure of around $\$ 218$. However, overall, pain medication costs did not contribute significantly to the total medication costs for all 3 disease groups. Instead, for patients with $\mathrm{pDPN}$, the most frequently used therapeutic groups of medications with proportions of users and weighted average annual costs within 5 years of index event were as follows: Central Nervous System (99.8\%, \$1218.9), Cardiovascular Agents (94.8\%, \$858.3), Hormones and Synthetic Substitutes (94.0\%, \$2468.2), and Anti-infective Agents (86.3\%, $\$ 181.1)$. These medications and associated costs are similar when compared with nonpainful DPN, except for a large reduction in Central Nervous System (35.4\%, \$131.0). The detailed proportions and weighted average annual costs within 5 years of index event are Cardiovascular Agents (85.3\%, \$724.9), Hormones and Synthetic Substitutes (83.1\%, \$2011.1), and Anti-infective Agents (64.9\%, \$79.8). In DM controls, the top therapeutic groups with proportions of users and weighted average annual costs within 5 years of index are Central Nervous System (83.3\%, \$709.9), Cardiovascular Agents (80.2\%, \$597.6), Anti-infective Agents $(79.8 \%, \$ 148.1)$, and Hormones and Synthetic Substitutes $(72.5 \%, \$ 479.8)$.

\section{Opioid use among patients with pDPN}

We further analyzed healthcare costs of patients with pDPN, comparing those who used opioids within 1 year of index dates vs those who did not (table e-2, links.lww.com/ $\mathrm{CPJ} / \mathrm{A} 103)$. Our results indicate significantly higher baseline and 1-year postdiagnosis costs associated with opioid use. At baseline, pDPN opioid users' associated costs were $\$ 14,429$ compared with $\$ 11,238$ for nonusers. These subsequently increased within a year to $\$ 19,091$ and $\$ 12,003$, respectively.

In addition, we found that 113,116 or $43.6 \%$ of DM controls had used opioids within 1 year of diagnosis compared with 51,785 or $61.6 \%$ of patients with pDPN. Patients with nonpainful DPN, by definition, did not use opioids during this time frame. Table e-3 (links.lww.com/CPJ/A103) further shows the temporal change in opioid use from 2010 to 2015 , indicating that the prevalence of opioid use peaked for patients diagnosed in 2011, but subsequently dropped. By comparison, an estimated 2 million

\section{Our results indicate significantly} higher baseline and 1-year postdiagnosis costs associated with opioid use. 
Table 2 Total inpatient and outpatient costs and medication-related costs

\begin{tabular}{|c|c|c|c|c|}
\hline & Diabetic control $(\mathrm{N}=259223)$ & Nonpainful DPN (N = 17267) & pDPN (N = 84069) & Total (N = 360559) \\
\hline \multicolumn{5}{|l|}{ Baseline year } \\
\hline $\mathbf{N}$ & 259223 & 17267 & 84069 & 360559 \\
\hline Mean (SD) & $11182.3(17219.0)$ & $11906.1(17649.6)$ & $19393.0(24200.7)$ & $13131.4(19405.7)$ \\
\hline Median (Q1, Q3) & $6382.3(2645.6,13369.8)$ & $7715.5(3788.2,13915.4)$ & $13448.7(7324.8,23400.6)$ & $7867.4(3339.4,15984.1)$ \\
\hline \multicolumn{5}{|c|}{ First year after index } \\
\hline $\mathbf{N}$ & 259223 & 17267 & 84069 & 360559 \\
\hline Mean (SD) & $14815.8(21746.5)$ & $13735.7(19855.3)$ & 24429.8 (29503.9) & $17005.7(24055.1)$ \\
\hline Median (Q1, Q3) & $8825.2(4218.4,17408.4)$ & $9036.6(4759.5,15987.0)$ & $16795.3(9537.1,28899.9)$ & $10447.4(5026.4,20230.2)$ \\
\hline \multicolumn{5}{|c|}{ Second year after index } \\
\hline $\mathbf{N}$ & 179515 & 12033 & 58742 & 250290 \\
\hline Mean (SD) & $12095.7(17970.2)$ & $14201.8(22037.5)$ & $20510.4(26615.0)$ & $14171.8(20826.1)$ \\
\hline Median (Q1, Q3) & $7041.1(3140.2,14355.3)$ & $8945.3(4227.0,16799.7)$ & $13867.8(7261.7,24711.4)$ & $8468.9(3773.3,17079.9)$ \\
\hline \multicolumn{5}{|c|}{ Third year after index } \\
\hline $\mathbf{N}$ & 87097 & 5213 & 28667 & 120977 \\
\hline Mean (SD) & $11351.3(16729.3)$ & $13334.7(21842.2)$ & $19672.1(23578.8)$ & $13408.5(19134.4)$ \\
\hline Median (Q1, Q3) & $6708.2(2849.5,13803.2)$ & $8594.3(3982.1,15810.0)$ & $13576.0(7051.7,24247.9)$ & $8128.2(3493.4,16559.6)$ \\
\hline \multicolumn{5}{|c|}{ Forth year after index } \\
\hline $\mathbf{N}$ & 52687 & 2724 & 16417 & 71828 \\
\hline Mean (SD) & $11058.3(15817.5)$ & $13881.5(18172.5)$ & $19902.0(22365.8)$ & $13186.7(18000.4)$ \\
\hline Median (Q1, Q3) & $6558.3(2722.6,13504.4)$ & $8783.7(4072.0,16912.9)$ & $13974.9(7002.1,25029.3)$ & $7934.8(3348.7,16451.6)$ \\
\hline \multicolumn{5}{|c|}{ Fifth year after index } \\
\hline $\mathbf{N}$ & 26256 & 1184 & 7185 & 34625 \\
\hline Mean (SD) & $11507.4(15959.1)$ & $14708.2(20953.0)$ & $21546.0(25036.3)$ & $13699.9(18832.0)$ \\
\hline Median (Q1, Q3) & $6995.0(2916.9,14103.6)$ & $9309.8(4365.2,17606.0)$ & $14857.1(7726.3,26872.2)$ & $8351.6(3508.4,16876.5)$ \\
\hline
\end{tabular}

Abbreviations: DPN = diabetic peripheral neuropathy; pDPN = painful diabetic peripheral neuropathy.

individuals in the United States have opioid use disorder associated with prescription opioids. ${ }^{11}$

\section{Regression model}

The adjusted cost ratios with $95 \%$ confidence intervals (CIs) and $p$ values were calculated using a GEE regression model. Overall, the pDPN cohort was associated with total median costs that were approximately $16 \%-31 \%$ higher than DM controls throughout our study time frame. At baseline, pDPN costs were $20 \%$ higher than DM controls (95\% CI [1.19, 1.21], $p<0.001)$. This difference dropped to $16 \%$ in the 1 st year after diagnosis (95\% CI [1.15, 1.17], $p<0.001$ ), but increased gradually over time to up to $31 \%$ in the 5 th year $(95 \%$ CI [1.27, $1.34], p<0.001)$. Controlling for other covariates, patients with nonpainful DPN accrued less total median costs than DM controls by $24 \%$ at baseline ( $95 \%$ CI $[0.75,0.78], p<0.001$ ), while the difference in costs shrunk over time to $6 \%$ in the 5 th year of diagnosis $(95 \% \mathrm{CI}[0.87,1.02], p=0.1242)$, which was not a significant difference thereafter.

\section{Pain medication use}

The breakdown of pain medication usage over a 5-year period was based on 3 classes of medication: opioids, anticonvulsant drugs (AEDs), and antidepressants. In general, pain medications were more commonly used by patients with pDPN. Among them, 71.7\% had at least 1 prescription of opioids, $51.1 \%$ used AEDs, and $57.1 \%$ had 1 or more prescriptions for antidepressants postindex date. The unadjusted odds ratios (ORs) indicated that compared with DM controls, patients with pDPN were $200 \%, 356 \%$, and $224 \%$ more likely to use opioids, AEDs, and antidepressants, respectively. 
Figure 2 Total inpatient and outpatient and medication cost time trend

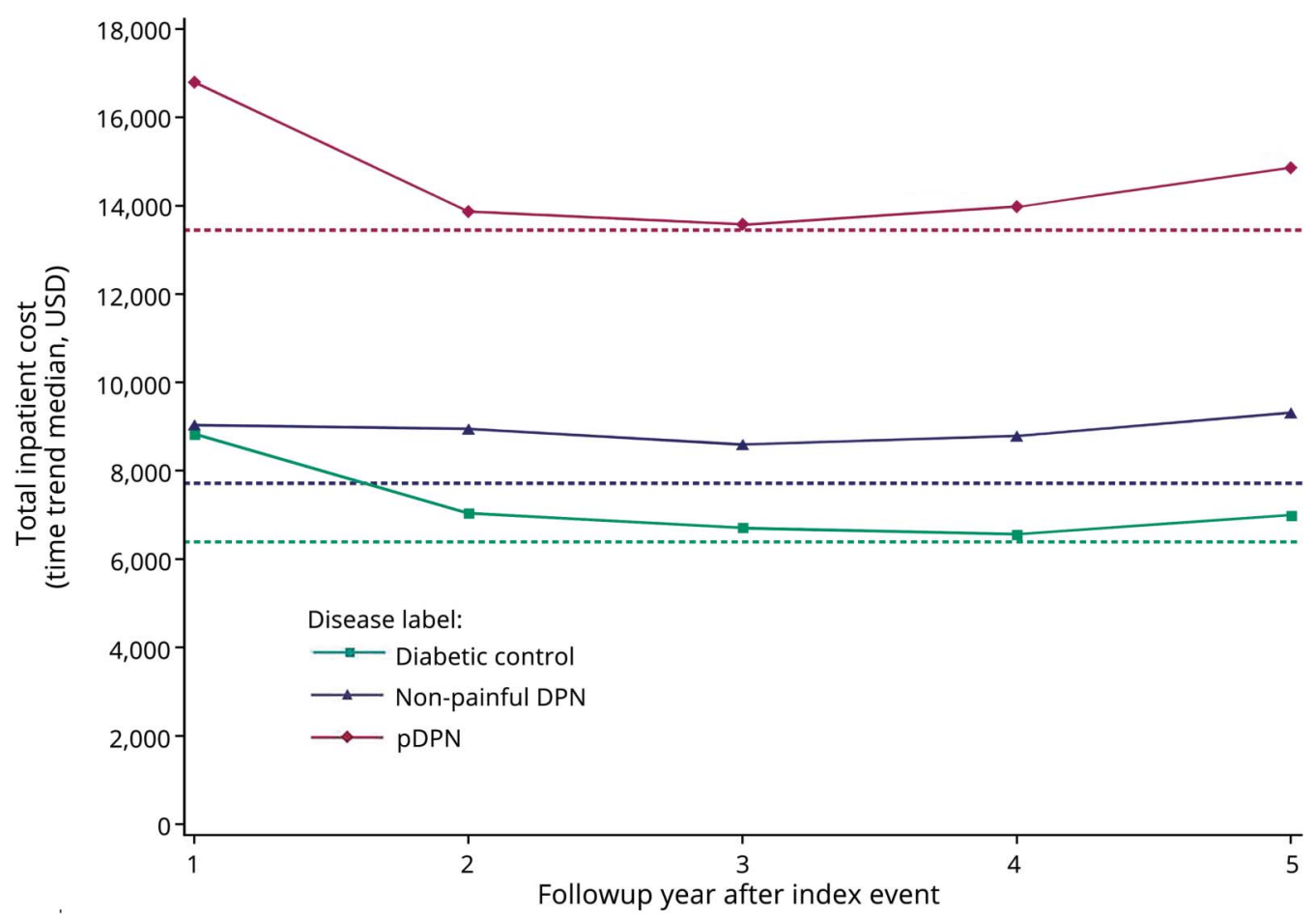

2010-2015 outcome time trend (total inpatient/outpatient costs and medication-related costs) for pDPN, nonpainful DPN, and diabetic controls. Dashed lines represent baseline costs. DPN = diabetic peripheral neuropathy; pDPN = painful diabetic peripheral neuropathy.

Only $18.2 \%$ of patients with nonpainful DPN used opioids, $5.7 \%$ had prescriptions for AEDs, and $5.5 \%$ had 1 or more prescriptions for antidepressants. This contrasted sharply with DM controls, where $56.3 \%$ used narcotics, $22.7 \%$ AEDs, and $37.3 \%$ antidepressants.

\section{Downstream complications}

Within 1 year of diagnosis, $1.6 \%$ of patients with $\mathrm{pDPN}$ had undergone lower limb amputations, whereas only $0.1 \%$ of DM controls and $0.7 \%$ of patients with nonpainful DPN had had the same procedure. Among those following up for at least 2 years, the proportion of patients undergoing lower limb amputations increased to $2.2 \%$ for $\mathrm{pDPN}, 0.2 \%$ for DM controls, and $1.3 \%$ among patients with nonpainful DPN. The risk of a lower limb amputation was 16.24 times that of DM controls within a year of diagnosis (95\% CI $[2.15,122.72], p=0.0003)$ and 11.22 times within 2 years $(95 \%$ CI $[2.63,47.86], p<0.0001)$. There was no statistically significant difference in the risk of amputations between patients with pDPN and nonpainful DPN (95\% CI $[0.94,5.63], p=0.0913)$ or between patients with nonpainful DPN and DM controls (95\% CI [0.86, 57.34], $p=0.0698$ ) within 1 year of diagnosis. However, within 2 years of diagnosis, the nonpainful DPN group had a significantly higher risk of lower limb amputations than DM controls (OR 6.57, 95\% CI [1.48, 29.20], $p=0.0072$ ).
In addition, the incidence of lower extremity infections (e.g., cellulitis and osteomyelitis) was much higher for all 3 disease groups compared with lower limb amputations. Among patients with pDPN, 16.1\% had lower extremity infections within a year of diagnosis, which is higher than the $9.3 \%$ incidence of DM controls (OR 1.87, 95\% CI $[1.43,2.46], p<0.0001)$. Within 2 years of diagnosis, approximately one-fourth of patients with pDPN (23.4\%) had experienced lower extremity infections. The odds of lower extremity infections for patients with pDPN were approximately the same when compared with diabetic controls if the follow-up time is extended to 2 years (OR $1.86,95 \%$ CI $[1.48,2.34], p<0.0001)$. In comparison, $9.3 \%$ of diabetic controls and $8.3 \%$ of patients with nonpainful DPN had lower extremity infections within 1 year of diagnosis; these numbers rose to $14.1 \%$ for diabetic controls and $12.9 \%$ for patients with nonpainful DPN at the 2-year mark.

The incidence of falls and fall-related injuries also tended to be higher in the pDPN cohort compared with the nonpainful DPN and control patients with diabetes. Within a year of diagnosis, a total of 1578 patients ( $0.4 \%$ of the entire cohort) had falls or fall-related injuries, $583(0.7 \%)$ of which were patients with pDPN compared with 61 nonpainful DPN $(0.4 \%)$ and 934 diabetic controls $(0.4 \%)$. Within 2 years of diagnosis, these numbers had increased substantially such 
Table 3 Relative incidence of nonmedical management in patients with PDN at 1-year follow-up

\begin{tabular}{llll}
\hline & Diabetic control $(\mathrm{N}=259223)$ & Nonpainful DPN $(\mathrm{N}=17267)$ & $\mathrm{pDPN}(\mathrm{N}=84069)$ \\
\hline Psychotherapy, individual & $18827(7.3 \%)$ & $316(1.8 \%)$ & $6850(8.1 \%)$ \\
\hline Psychotherapy, family & $1363(0.5 \%)$ & $22(0.1 \%)$ & $398(0.5 \%)$ \\
\hline Psychotherapy, group & $888(0.3 \%)$ & $13(0.1 \%)$ & $345(0.4 \%)$ \\
\hline Therapeutic psychological services & $4523(1.7 \%)$ & $51(0.3 \%)$ & $1793(2.1 \%)$ \\
\hline $\begin{array}{l}\text { Physical medicine } \\
\text { Chiropractic services }\end{array}$ & $66958(25.8 \%)$ & $3402(19.7 \%)$ & $28815(34.3 \%)$ \\
\hline $\begin{array}{l}\text { Home health physical, occupational, and } \\
\text { speech therapy }\end{array}$ & $6220(2.4 \%)$ & $1067(6.2 \%)$ & $6219(7.4 \%)$ \\
\hline
\end{tabular}

Abbreviations: DPN = diabetic peripheral neuropathy; pDPN = painful diabetic peripheral neuropathy.

that $2.2 \%$ of patients with pDPN had had falls and fall-related injuries compared with $1.5 \%$ of nonpainful DPN and $1.1 \%$ of diabetic controls.

\section{Nonmedical management of pDPN}

The incidence rates of nonmedical treatments for the 3 disease groups are summarized for those with at least 1 year (table 3 ) and 2 years (table 4 ) of follow-up. In general, more patients with pDPN used nonmedical management of any kind than DM controls, except for chiropractic services. For patients with $\mathrm{pDPN}$, the most common were psychotherapy (individual; 10.6\%), physical medicine (46.6\%), and chiropractic services $(9.8 \%)$ within 2 years of follow-up.

\section{Discussion}

This study serves as a substantial and recent economic analysis of painful diabetic neuropathy. Expanding the scope of this study beyond health care economics and more directly into clinical outcomes, we further studied the incidence of downstream complications and use of nonmedical management for symptomatic relief in $\mathrm{pDPN}$.
Evidence for sustained elevated costs of pDPN over time can be seen in our temporal plots, which showed that patients with pDPN spent about twice as much as DM controls annually until the 5 th year of diagnosis. The highest median costs for the pDPN cohort were approximately $\$ 16,795$ (or $\$ 7066$ more than diabetic controls) occurring in the 1st year after diagnosis, pointing to worse short-term morbidity and economic costs for pDPN after diagnosis. In addition, our regression model suggested that even after adjusting for confounders, patients with pDPN continued to spend more than DM controls in the 1st year after diagnosis, with the gap widening over time. These results point to clear differences in economic costs among patients with DM and those with additional painful neuropathic manifestations, providing an impetus for clinicians to work together with patients to help treat early and reduce disease progression.

In addition, we found that around $33 \%$ of the total costs associated with pDPN, compared with $20.1 \%$ for DM controls, were related to medications from outpatient pharmacies. That $71.7 \%$ of patients with pDPN have at least 1 opioid prescription after diagnosis, $51.1 \%$ have used AEDs, and $57.1 \%$ have used antidepressants is further evidence of the

Table 4 Relative incidence of nonmedical management in patients with PDN at 2-year follow-up

\begin{tabular}{|c|c|c|c|}
\hline & Diabetic control $(\mathrm{N}=179515)$ & Nonpainful DPN (N = 12033) & $\operatorname{pDPN}(\mathrm{N}=58742)$ \\
\hline Psychotherapy, individual & $17099(9.5 \%)$ & 367 (3.0\%) & $6220(10.6 \%)$ \\
\hline Psychotherapy, family & $1494(0.8 \%)$ & $23(0.2 \%)$ & $428(0.7 \%)$ \\
\hline Psychotherapy, group & $944(0.5 \%)$ & $14(0.1 \%)$ & $342(0.6 \%)$ \\
\hline Therapeutic psychological services & $4460(2.5 \%)$ & $62(0.5 \%)$ & $1758(3.0 \%)$ \\
\hline Physical medicine & $65350(36.4 \%)$ & 3721 (30.9\%) & $27369(46.6 \%)$ \\
\hline Chiropractic services & $22682(12.6 \%)$ & $953(7.9 \%)$ & $5742(9.8 \%)$ \\
\hline $\begin{array}{l}\text { Home health physical, occupational, and } \\
\text { speech therapy }\end{array}$ & $6238(3.5 \%)$ & $372(3.1 \%)$ & $3956(6.7 \%)$ \\
\hline
\end{tabular}

Abbreviations: DPN = diabetic peripheral neuropathy; pDPN = painful diabetic peripheral neuropathy. 
intrinsic difficulty in finding the right medications that work for $\mathrm{pDPN}$ symptoms and the various associated comorbidities associated with the disease. At the same time, the relatively high use of pain medications in the DM control group suggests a likely diagnostic coding error for patients with painful DPN with underestimation of this cohort's prevalence and subsequent HCRU. It is important to consider, however, that such patients might have other etiologies (nociceptive, parietal, etc.) of pain that are unrelated to diabetes.

The relative prevalence of $\mathrm{pDPN}$ in our study was $23.3 \%$, whereas nonpainful DPN constituted only $4.8 \%$ of the cohort. Although the prevalence of $\mathrm{pDPN}$ found in our study is similar to recent population-based surveys, ${ }^{12}$ the relatively low prevalence of nonpainful DPN suggests that many of these patients were inappropriately diagnosed as DM without neuropathy (although it might also be that many patients with neuropathy choose to not take pain medications). As a result, our subsequent HCRU analyses might have underestimated the incremental costs and health impact associated with patients with painful and nonpainful DPN. Systematic reviews have additionally focused on the "humanistic" burden of pDPN that is less tangible and more difficult to study in large database analyses, including limitations in general functioning, reduced sleep, mood disorders, and worsening productivity. ${ }^{13}$ Our study was, of course, limited in eliciting these. Furthermore, clinicians are beginning to realize that women experience disproportionately more pain and associated distress than men, ${ }^{14}$ an insight that might shed light on our patient demographics, where patients with $\mathrm{pDPN}$ tended to have a slight female predominance, but nonpainful DPN consisted clearly of more men. Also, as expected due to the natural progression of disease, patients with $\mathrm{pDPN}$ had a higher proportion of elderly patients with Medicare insurance than DM controls.

In comparison, the previous largest cohort of patients with pDPN analyzed for HCRU against DM controls consisted of 323,378 patients isolated from the Humedica database. ${ }^{15}$ Here, an additional tier for severe pDPN (based on $>7 / 10$ pain scores within 15 days of being diagnosed with diabetic neuropathy) was considered. Such a stratification was supplemented by a detailed breakdown of HCRU, delineating costs for specific items such as foot examinations and emergency department visits, both of which were admittedly missing from our study. Ultimately, the Humedica study reported 1-year costs that ranged from $\$ 6,632$ in DM controls to $\$ 30,755$ for those with severe $\mathrm{pDPN}$, a range much wider than ours $(\$ 8,852-\$ 16,795)$ for the same period. Importantly, our work attempts to compare these costs with their prediagnosis baselines and illustrate how such costs change over time. Moreover, our analysis provides the additional rigor of a multivariable regression model, allowing for firmer conclusions regarding the true health care economics of this important diabetic complication.

Other complications of DM and pDPN were also reported and analyzed. The odds of 2-year risk of lower limb amputations in patients with $\mathrm{pDPN}$ were 10 times higher than diabetic controls, a result that indicates improved outcomes compared with findings from the last century, where a 15 -fold increased risk in patients with diabetes has been reported. ${ }^{16,17}$ In addition, the odds of 1-year lower extremity infection in patients with $\mathrm{pDPN}$ are $86 \%$ more than DM controls. A substantial number of patients, especially those diagnosed with pDPN, had experienced subsequent falls and fall-related injuries. Although we only analyzed up to 2 years postdiagnosis, the incidence and prevalence of these complications are likely to rise with time. Reduced pain and trauma sensitivity with progression of the neuropathy might explain these findings.

As an indicator of the difficulties inherent in disease management, we also obtained an understanding of nonmedical therapies used by patients. It is now evident that long-term opioid treatment alone is often ineffective and, in many cases, unsafe for the treatment of chronic pain. A multidisciplinary treatment strategy is now advocated that shifts the clinical paradigm away from heavy reliance on opioids to a treatment approach that incorporates a diverse array of nonpharmacologic pain treatment modalities (NPMs). Multiple studies have demonstrated the effectiveness of NPMs in improving chronic pain outcomes, including physical therapy, cognitive behavioral therapy, mindfulness-based stress reduction, yoga, chiropractic treatment, and a variety of interventional approaches. ${ }^{18}$ Each of these nonopiate solutions may have fewer long-term adverse side effects than long-term opiates. Patients and physicians have noted that common barriers to nonopiate solutions include barriers to access, including cost, insurance coverage, scheduling, and availability of resources. Indeed, in our study, among those with at least 2 years of follow-up data, $46.6 \%$ of patients with pDPN had used physical medicine, and up to $10 \%$ had used psychotherapy (individual) and chiropractic services. In interpreting these results, it is also important to consider the likely high rates of affective disorders (including depression and anxiety) in such patients experiencing chronic disease, which can account for their use of psychotherapy, antidepressants, and other treatments.

Our study was limited in several ways. Medication costs were captured by relying solely on outpatient pharmacy claims due to incomplete access to the MarketScan inpatient medication database. We used therapeutic classes 60 and 61 to define opioid medications. However, the 2 therapeutic classes might not cover all types of opioid medications and could possibly result in underestimation of the sample size of patients with pDPN. Also, although CNS drugs (especially, opioid agonists, antidepressants, and anticonvulsants) account for the largest difference in medication costs between the pDPN and nonpainful DPN cohorts, we were limited by our database in ascertaining whether these drugs were actually being prescribed for neuropathic pain or other diagnoses. In addition, although we used a 1-month washout period to reduce the influence of escalating hospital visits and diagnostic tests before a DM or DPN diagnosis was made, our exploratory analyses failed to indicate what exactly explained the high HCRU associated with pDPN and the other cohorts. In our longitudinal analysis, the sample size decreased every year because of 
insufficient follow-up. Also, because the diagnosis index dates differed between the pDPN and DM controls, our results might have been biased toward greater health care costs and complications for the pDPN cohort. Furthermore, because diabetic complications, due to the chronic nature of the disease, usually become worse with time, the difference in index dates might explain the age differential between the 2 cohorts and at least some of the increase in health costs and other negative outcomes. Withstanding other limitations of a retrospective investigation, our study was able to review and quantify the HCRU, associated complications, and nonmedical management of painful diabetic neuropathy. We provided insights into the temporal changes in health care economics associated with pDPN and provided a glimpse into the potential long-term complications and nonmedical interventions experienced and sought by patients, areas that are becoming more relevant with the expansion of nonopiate treatments for $\mathrm{pDPN} .{ }^{19,20}$

Diabetes, unfortunately, remains a financially and personally costly disease, both at the individual and societal level. Prevention and treatment of diabetes and its early complications will be essential to reducing these costs.

\section{Study funding}

Supported by the NIH (UL1TR002553).

\section{Disclosure}

The authors report no disclosures relevant to the manuscript. Full disclosure form information provided by the authors is available with the full text of this article at Neurology.org/cp.

\section{Publication history}

Received by Neurology: Clinical Practice November 14, 2018. Accepted in final form April 8, 2019.

\section{Appendix Authors}

\begin{tabular}{lll}
\hline Name & Location & Contribution \\
\hline Musa Kiyani, BS & $\begin{array}{l}\text { Duke University Medical } \\
\text { Center (DUMC) }\end{array}$ & $\begin{array}{l}\text { Designed and } \\
\text { conceptualized the study, } \\
\text { interpreted the data, and } \\
\text { drafted the manuscript }\end{array}$ \\
\hline $\begin{array}{l}\text { Zidanyue Yang, } \\
\text { MB }\end{array}$ & DUMC & $\begin{array}{l}\text { Acquired and analyzed the } \\
\text { data and revised the } \\
\text { manuscript }\end{array}$ \\
\hline $\begin{array}{l}\text { Lefko T. } \\
\text { Charalambous, }\end{array}$ & DUMC & $\begin{array}{l}\text { Interpreted the data and } \\
\text { revised the manuscript }\end{array}$ \\
\hline Syed M. Adil, BS & DUMC & $\begin{array}{l}\text { Interpreted the data and } \\
\text { revised the manuscript }\end{array}$ \\
\hline Hui-Jie Lee, PhD & DUMC & $\begin{array}{l}\text { Acquired and analyzed the } \\
\text { data and revised the } \\
\text { manuscript }\end{array}$ \\
\hline
\end{tabular}

\section{Appendix (continued)}

\begin{tabular}{lll}
\hline Name & Location & Contribution \\
\hline Siyun Yang, MS & DUMC & $\begin{array}{l}\text { Acquired and analyzed the } \\
\text { data and revised the } \\
\text { manuscript }\end{array}$ \\
\hline $\begin{array}{l}\text { Promila } \\
\text { Pagadala, PhD }\end{array}$ & DUMC & $\begin{array}{l}\text { Interpreted the data and } \\
\text { revised the manuscript }\end{array}$ \\
\hline $\begin{array}{l}\text { Beth Parente, PA- } \\
\text { C, MHS }\end{array}$ & DUMC & $\begin{array}{l}\text { Interpreted the data and } \\
\text { revised the manuscript }\end{array}$ \\
\hline $\begin{array}{l}\text { Susan Spratt, MD } \\
\text { DUMC }\end{array}$ & $\begin{array}{l}\text { Interpreted the data and } \\
\text { revised the manuscript }\end{array}$ \\
\hline $\begin{array}{l}\text { Shivanand P. Lad, } \\
\text { MD, PhD }\end{array}$ & DUMC & $\begin{array}{l}\text { Interpreted the data and } \\
\text { revised the manuscript }\end{array}$ \\
\hline
\end{tabular}

\section{References}

1. Boulton AJ, Vileikyte L, Ragnarson-Tennvall G, Apelqvist J. The global burden of diabetic foot disease. Lancet 2005;366:1719-1724.

2. Pirart J. Diabetes mellitus and its degenerative complications: a prospective study of 4,400 patients observed between 1947 and 1973. Diabetes Care 1978;1: $168-252$.

3. Abbott CA, Malik RA, van Ross ER, Kulkarni J, Boulton AJ. Prevalence and characteristics of painful diabetic neuropathy in a large community-based diabetic population in the UK. Diabetes Care 2011;34:2220-2224.

4. Fields HL. Painful dysfunction of the nervous system. In: Fields HL, editor. Pain. New York: McGraw-Hill Information Services Co; 1987:133-169.

5. Backonja M, Beydoun A, Edwards KR, et al; Gabapentin Diabetic Neuropathy Study Group. Gabapentin for the symptomatic treatment of painful neuropathy in patients with diabetes mellitus: a randomized controlled trial. JAMA 1998;280: 1831-1836.

6. Calissi PT, Jaber LA. Peripheral diabetic neuropathy: current concepts in treatment. Ann Pharmacother 1995;29:769-777.

7. Berger A, Dukes EM, Oster G. Clinical characteristics and economic costs of patients with painful neuropathic disorders. J Pain 2004;5:143-149.

8. Dworkin RH, Malone DC, Panarites CJ, Armstrong EP, Pham SV. Impact of postherpetic neuralgia and painful diabetic peripheral neuropathy on health care costs. J Pain 2010;11:360-368.

9. Charlson ME, Pompei P, Ales KL, MacKenzie CR. A new method of classifying prognostic comorbidity in longitudinal studies: development and validation. J Chronic Dis 1987;40:373-383.

10. Deyo RA, Cherkin DC, Ciol MA. Adapting a clinical comorbidity index for use with ICD-9-CM administrative databases. J Clin Epidemiol 1992;45: 613-619.

11. Schuchat A, Houry D, Guy GP. New data on opioid use and prescribing in the United States. JAMA 2017;318:425-426.

12. Davies M, Brophy S, Williams R, Taylor A. The prevalence, severity, and impact of painful diabetic peripheral neuropathy in type 2 diabetes. Diabetes Care 2006;29: $1518-1522$.

13. Alleman CJ, Westerhout KY, Hensen M, et al. Humanistic and economic burden of painful diabetic peripheral neuropathy in Europe: a review of the literature. Diabetes Res Clin Pract 2015;109:215-225.

14. Paller CJ, Campbell CM, Edwards RR, Dobs AS. Sex-based differences in pain perception and treatment. Pain Med 2009;10:289-299.

15. Sadosky A, Mardekian J, Parsons B, Hopps M, Bienen EJ, Markman J. Healthcare utilization and costs in diabetes relative to the clinical spectrum of painful diabetic peripheral neuropathy. J Diabetes Complicat 2015;29:212-217.

16. Bild DE, Selby JV, Sinnock P, Browner WS, Braveman P, Showstack JA. Lowerextremity amputation in people with diabetes: epidemiology and prevention. Diabetes Care 1989;12:24-31.

17. Most RS, Sinnock P. The epidemiology of lower extremity amputations in diabetic individuals. Diabetes Care 1983;6:87-91.

18. Brunelli B, Gorson KC. The use of complementary and alternative medicines by patients with peripheral neuropathy. J Neurol Sci 2004;218:59-66.

19. de Vos CC, Meier K, Zaalberg PB, et al. Spinal cord stimulation in patients with painful diabetic neuropathy: a multicentre randomized clinical trial. Pain 2014;155: 2426-2431.

20. van Beek M, Slangen R, Schaper NC, et al. Sustained treatment effect of spinal cord stimulation in painful diabetic peripheral neuropathy: 24-month follow-up of a prospective two-center randomized controlled trial. Diabetes Care 2015;38: e132-e134 